\title{
Negovsky y Safar unidos por la ciencia de la reanimación
}

\section{Negovsky and Safar together for science of resuscitation}

José Ricardo Navarro-Vargas', Natalia Botero-Jaramillo², María Fernanda Bueno², Karina Varela-Macuit|²

\begin{abstract}
Objective: This article characterize the contributions of Peter Safar and Vladimir Negovsky to the field of resuscitation and critical medicine during the 20th century and the dawn of the 21st century with the purpouse to correlate the discoveries and developments of each one, with their life stories and with the historical moments throughout the 20th century. Materials and Methods: A review of literature on the subject was conducted, in which primary and secondary sources on the life and scientific and academic production of Vladimir Negovsky and Peter Safar were searched in online databases, in English as well as Spanish. Results: Peter Safar and Vladimir Negovsky, although they had two very different life histories and developed their academic contributions in opposite political contexts, this did not prevent each of them from laying the foundations of cardiopulmonary resuscitation, both in the concepts and the physiopathological understanding of the damage processes, as well as the procedures to act in a timely manner, minimizing them. They also contributed to critical medicine, and to the formation of intensive care units. This work aims to show how a field of knowledge is based on collective work, as the discovery does not depend on a single character, but on a social, historical process, which is also based on the knowledge and elements developed by others.
\end{abstract}

\section{RESUMEN}

Objetivo: Con este artículo se caracterizan los aportes de Peter Safar y Vladimir Negovsky en el campo de la reanimatología y de la medicina crítica durante el siglo XX y albores del siglo XXI, y se correlacionan sus investigaciones y descubrimientos con sus historias de vida y momentos compartidos. Materiales y

\section{Key words:}

Anesthesiology, reanimatology, Safar, Negovsky, history

\section{Palabras clave:}

Anestesiología, reanimación, Safar, Negovsky, historia

\footnotetext{
Profesor de Medicina, Universidad Nacional de Colombia.

2 Estudiantes Pregrado de Medicina, Línea de Profundización Paro Cardiaco en el Adulto.
}

Fecha de recepción: 26 de enero de 2019

Fecha de aceptación: 08 de febrero de 2019

\section{ORCID}

https://orcid.org/0000-0003-2548-1325

\section{Correspondencia:}

Navarro-Vargas JR

Email: jrnavarrov@unal.edu.co 


\begin{abstract}
Métodos: Se realizó una revisión de literatura sobre el tema, mediante la búsqueda de fuentes primarias y secundarias sobre la vida y producción científica y académica de Vladimir Negovsky y Peter Safar, en bases de datos en línea, en inglés y en español. Resultados: Peter Safar y Vladimir Negovsky, pese a tener dos historias de vida muy distintas y desarrollar sus aportes académicos en contextos políticos diferentes, coincidieron en un campo de la ciencia que no les impidió que cada uno sentara las bases de la reanimación cardio cerebro pulmonar, tanto en los conceptos y en la comprensión fisiopatológica de los procesos de lesión, como en los procedimientos para prevenir la muerte y minimizar los efectos del proceso isquemia/reperfusión. Así mismo, concibieron y desarrollaron las unidades de medicina crítica y cuidado intensivo, el sistema integral para el transporte de los pacientes y el campo vasto en la formación de posgrados como anestesiología y medicina crítica. Conclusión: Definitivamente la ciencia no tiene fronteras políticas y se fundamenta en el trabajo colectivo. Estos dos personajes vivieron tiempos políticos y sociales adversos, unieron lazos de amistad y compartieron experiencias para producir cambios históricos en la ciencia de la reanimación conocida como Reanimatología.
\end{abstract}

\section{Introducción}

a ciencia médica ha tratado de explorar los eventos Lque ocurren alrededor de la muerte con el propósito de "salvar los corazones y los cerebros de aquellos individuos que aún son demasiado jóvenes para morir"[1].

Peter Safar de Austria y Vladimir Negovsky de la Unión Soviética han sido considerados los padres de la reanimación en el mundo. El primero, por dedicar todos sus esfuerzos a comprender los mecanismos de la circulación cardiaca y la respiración después de un paro cardiaco; el segundo por comprender los procesos fisiopatológicos que ocurren alrededor de la muerte. Se conocieron en el Primer Congreso Europeo de Anestesiología, en Viena, en septiembre de 1962[2],[3],[4] y pese a no compartir las mismas tendencias políticas, unieron esfuerzos para desarrollar la reanimación en el mundo. Safar produjo más de 1.400 publicaciones, entre las que se cuentan 384 artículos revisados por pares, más de 30 libros y manuales y más de 600 resúmenes; Negovsky fue autor y coautor en más de 300 publicaciones científicas y 14 monografías, en las que propuso una serie de estrategias para evitar la muerte ante la obstrucción coronaria fatal y minimizar los daños que produce el síndrome isquemia reperfusión, descrito por él como enfermedad posresucitación cardiaca[3],[4],[5],[6]. Estos dos investigadores promovieron la medicina de la paz, en un mundo transformado por la guerra y la división política, y fueron postulados al premio Nobel de Medicina en 3 oportunidades[3],[4],[5]. Safar con- sideraba a Negovsky el investigador más importante en la reanimatología[7], a pesar de ser él la persona con el mayor reconocimiento en la reanimación en el mundo occidental capitalista; se le considera el autor del acróstico ABC, DEF y GHI, en el cual demostraría la importancia de asociar las compresiones torácicas con la ventilación boca a boca en el entorno prehospitalario, hasta cuando la reanimación pasara a ser manejada por un equipo médico de emergencia $y$, finalmente, cuando el paciente saliera de la condición de paro cardiaco, y retornara a la circulación cardiaca espontánea, pudiera seguir el tratamiento en una unidad de cuidados especiales (las actuales UCI); Negovsky, desarrolló lo que hoy se conoce como el quinto eslabón de la cadena de sobrevida, el manejo integral posparo cardiaco, además, se le reconocen sus aportes sobre la agonía, la muerte clínica y biológica, y su fisiopatología[8],[9],[10],[11].

\section{Metodología}

El presente artículo de reflexión gira alrededor de las actividades científicas de dos personajes gigantes que revolucionaron la reanimación; se basó en una búsqueda de fuentes primarias y secundarias sobre los aportes de Peter Safar y Vladimir Negovsky en el campo de la anestesiología y de la reanimación, así como de algunos de los textos escritos fruto de sus investigaciones, que pudieran dar cuenta de aportes y propuestas, y de la relación que establecieron entre sí en el ámbito académico, y las motivaciones personales, 
sociales y políticas que dinamizaron sus producciones en investigación, gestión del conocimiento y creación de organizaciones, institutos, programas académicos y laboratorios de investigación entre otros.

Las fuentes fueron buscadas en línea, usando como descriptor el nombre de cada uno de los autores, tanto en inglés como en español. Se realizó una lectura de los resúmenes, identificando el contenido de los artículos, para seleccionar los más relevantes y que se ajustaran a los propósitos de esta reflexión. Los análisis se hicieron a la luz de los procesos históricos y sociopolíticos del siglo $X X$, las implicaciones personales como hombres de ciencia y ciudadanos con participación activa en la Segunda Guerra Mundial, la Guerra Fría, la Perestroika y la glásnost, así como el concepto de camaradería a medida que se iban desarrollando sus investigaciones[12],[13],[14].

\section{Resultados}

Encuentro de dos vidas interesantes para la humanidad y sus estudios en la reanimatología.

Peter Safar nació en Viena, Austria, el 12 de abril de 1924 en una familia de médicos. Su padre Karl fue oftalmólogo y su madre Vinca pediatra. Su abuelo fue editor médico cuya compañía se convirtió en uno de los brazos de Spinger Velag conocido como Minerva. El ambiente político en Europa estaba tenso por el Tercer Reich alemán, y especialmente en Viena por la inminencia de la ocupación nazi en 1938[3],[15].

Safar fue enviado a un campo de trabajo en Bavaria, lugar donde iba a ser integrado al ejército alemán de no haber sido por su ingenio, pues para que no fuera alistado en las filas militares, se frotó su cuerpo con tuberculina, generándose una gran reacción inflamatoria en su piel, siendo notificado por los médicos militares y exonerado. Ya para 1943, a la edad de 19 años, entró a la escuela médica en Viena, donde tuvo contacto directo con los fenómenos sicopatológicos de la guerra, pues asistió a personas que habían sufrido la indignidad y las secuelas de la guerra. Se graduó de médico el 19 de marzo de 1948. Un año más tarde, fue a la Universidad de Yale en Estados Unidos a hacer un entrenamiento en cirugía, hizo una breve pausa en 1950, en que retornó a Viena para casarse con Eva, a quien había conocido en 1947. Regresaron juntos a Estados Unidos y allí completó su residencia en anestesiología en la universidad de Pensilvania bajo las enseñanzas del doctor Robert $D$. Cripps entre 1950 y 1952[5],[15]. En este año tuvo que salir del país por temas migratorios, y se trasladó con su esposa a Perú, donde fundó uno de los prime- ros departamentos de anestesiología en Lima, en el Hospital Nacional de Cáncer. Los autores del presente artículo han mostrado mucho interés en indagar la motivación particular que tuvo Safar para trasladarse a Perú descartando cualquier otro país, y de esto es consciente la Sociedad Peruana de Anestesiología, Analgesia y Reanimación. Dos años más tarde regresó a Estados Unidos donde trabajó en el Hospital John Hopkins, allí fundó el Departamento Académico de Anestesiología e inició gran parte de sus investigaciones sobre la ventilación. En 1961 ingresó como docente a la Universidad de Pittsburgh, donde ayudó a establecer el primer programa de cuidados intensivos multidisciplinario[3],[5].

En 1966, un evento trágico ocurriría en su vida, que lo marcaría para siempre (y que lo llevó a dedicarse de manera obsesiva al estudio del rescate de la ventilación por personas inexpertas o legos en salud), la muerte de Elizabeth, su única hija mujer de tan solo 12 años de edad, quien desarrolló un estatus asmático severo que la llevó a un paro cardiaco y a la muerte. Este lamentable evento confirmaría la importancia de su trabajo y la necesidad de emprender acciones concretas que pudieran salvar vidas, no solo en el entorno hospitalario, sino en los hogares, sitios de trabajo y en las calles, donde las personas necesitarían una ayuda oportuna[3],[10]. Conformó un equipo de paramédicos y publicó su primer manual de resucitación cardiopulmonar, el cual se convertiría en un libro de texto fundamental para el aprendizaje de la reanimación cardiopulmonar[16],[17]. En la década de 1970 Safar creó un servicio de emergencia con la comunidad, contratando afroamericanos desempleados para entrenarlos en reanimación básica[3]. En 1978, renunció a la Universidad de Pittsburgh para concentrarse de lleno a la investigación en reanimación, y para ello, participó en la creación del Centro Internacional de investigaciones en Resucitación IRRC, encargado del estudio de paro cardiaco, shock, trauma, reanimatología y desastres donde estuvo hasta 1994, siendo remplazado por Patrick Kochanek, quien tuvo a bien denominar el instituto como "Centro Safar de Investigación en Reanimación"[3],[7].

Safar fue uno de los fundadores de la Sociedad Médica de Cuidados Críticos y, además de participar en la creación de un servicio de ambulancias de paramédicos, fue líder gremial de asociaciones profesionales como la de Médicos por la Responsabilidad Social y en la Asociación Internacional de Médicos para la Prevención de la Guerra Nuclear[9].

Se le consideró un humanista y hombre de ciencia que trató hasta sus últimos días de enseñarle a la comunidad las bases de la reanimación. Fue un hombre 
culto que tenía en la música uno de sus mayores pasatiempos, tocaba el piano y admiraba a músicos como Mahler y Bruckner, y disfrutaba y celebraba enormemente del vals, el cual le encantaba bailar y lo hacía muy bien.

Su vida siempre fue de retos, y al final tuvo que enfrentar un cáncer abdominal sometiéndose a cruentas cirugías y a la quimioterapia sin mayor éxito; falleció el 3 de agosto de 2003[7].

Vladimir Negovsky nació en la contraparte política del mundo capitalista occidental, el 19 de marzo de 1909 en Kozelt Chernigov, Ucrania, en una época en que el pueblo ruso aún no había vivido la revolución bolchevique. Sus padres fueron profesores y la infancia de Vladimir transcurrió en una finca con sus nueve hermanos. Cuando era niño salió con un amigo a caminar a través del hielo durante la celebración religiosa de domingo de Ramos, sufriendo una caída entre el hielo, siendo víctima de la hipotermia. Después de este episodio, sufrió de tuberculosis ósea, pasó un año en el hospital, se sometió a siete cirugías y pudo recuperar su movilidad con muletas, las cuales fue dejando poco a poco. Su contacto prematuro con el hospital y la cercanía a la muerte, lo llevarían a elegir la medicina como su profesión. Ingresó al Segundo Instituto Médico de Moscú en 1928 graduándose como médico en 1933. En 1934 ingresaría como investigador al Instituto de Transfusión de Sangre, bajo la tutoría del profesor Serguéy Bryukhonenko, creador del aparato de circulación de sangre artificial. Dos años más tarde él y otros investigadores más afines a sus ideas fueron despedidos del instituto, por lo que solicitaron al Consejo de Comisarios del Pueblo de la Unión Soviética, apoyo a la investigación sobre revitalización del cuerpo la cual le daría las bases científicas para la reanimación, bajo el principio de restaurar los procesos de vida en fenómenos similares a la muerte. Esto sería el inicio del primer laboratorio en el mundo en medicina de la reanimación, que en un principio estuvo adscrito al Instituto de Neurocirugía dirigido por un reconocido neurocirujano ruso Nikolay Nilovich Burdenko en 1936. Para 1948 el laboratorio se convertiría en una institución científica independiente, llamado Laboratorio de Investigación en Reanimación General de la Academia de Ciencias Médicas de la URSS, el cual sería, posteriormente, nombrado Instituto de Reanimatología de la Unión Soviética[4],[9],[18].

La Segunda Guerra Mundial cercaba al mundo bajo la inclemencia de los fusiles; fue llamada por los rusos la Gran Guerra Patriótica; estos lucharían en contra de la Alemania nazi, que tomó como punto de partida la invasión nazi a la Unión Soviética el 22 de junio de 1941, y como fin, la caída de Berlín a manos del Ejército Rojo el 3 de mayo de 1945. En dicho contexto, Negovsky llevaría a la práctica algunos de sus resultados, participando en una brigada del ejército en 1943, en la que trabajó en la primera línea del frente, atendiendo a heridos que estaban en agonía, en muerte clínica según su terminología, pero que aún podían ser revividos. Quería demostrar que el último latido del corazón no era necesariamente sinónimo de muerte, que él lo podía revertir. Su primer soldado atendido fue Valentin Cherepanov seguido de muchos otros, incluyendo al físico ruso que habló sobre la teoría de la relatividad Lev Landau, premio Nobel de Física en 1962, y quien sufrió un accidente automovilístico con compromiso craneano severo[6],[18].

El laboratorio sería uno de los escenarios centrales de Negovsky (Figura 1), donde propondría los principios de la reanimación enfocándose en las variables fisiológicas del corazón, los pulmones y el cerebro, en la lucha contra la muerte, tema que ocupó su pasión desde la experiencia de su formación académica y de su vida profesional. La posición de Negovsky sobre el papel del cerebro en el proceso de morir fue central en su obra. De ahí que el último latido del corazón no era para él, el fin de la vida como comúnmente se creía, pues el organismo permanecía por varios minutos en un estado reversible, en términos de recuperación del sistema nervioso, pudiendo ser retornado al estado vital. Para Negovsky lo que está acorde con

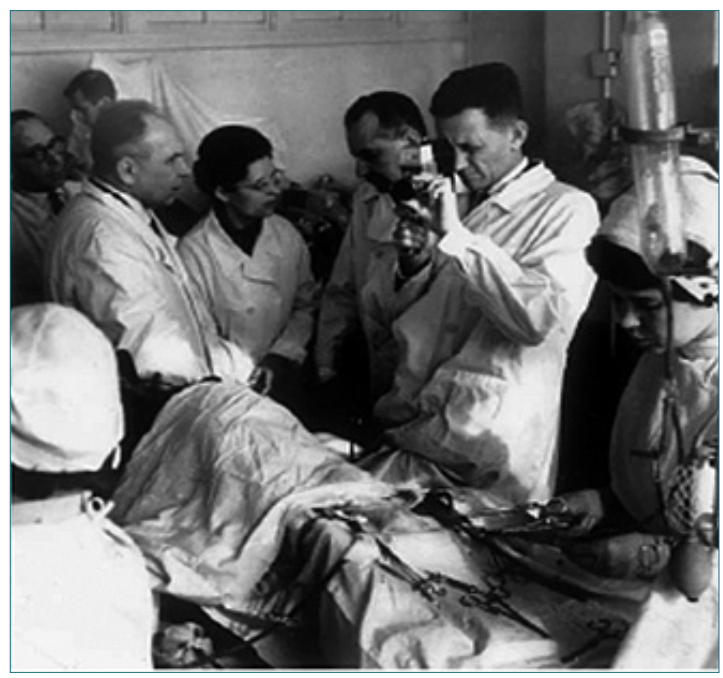

Figura 1. Negovsky (izquierda) y Safar (derecha). Fotografía tomada durante un experimento en que se hizo una exanguinación en el contexto de una resucitación en un paro cardiaco a un perro. En esta misma foto se encuentra Hugh Rosomoff (centro) en el laboratorio de Negovsky[4]. 
la vida es la existencia de signos de vitalidad cerebral y de allí su profundo interés por la patogenia de los estados terminales, y del avivamiento de los organismos[18]. En 1943 su tesis doctoral se basó sobre la fisiopatología y reversión de la muerte, la cual llegó a ser su primer libro traducido al inglés, y el insumo para ocho artículos más publicados en la Revista Americana de Medicina Soviética (American Review of Soviet Medicine)[4],[18]. Al finalizar la guerra fue promovido a profesor universitario, con la posibilidad de dar mayor difusión a su conocimiento y a los avances que en su laboratorio se estaban adelantando, con años de ventaja sobre los que se llevaban a cabo en países occidentales. Tal fue el caso del uso de compresiones torácicas externas y terapia eléctrica, que se practicaban en el laboratorio de Negovsky diez años antes que esto se redescubriera y se introdujera en los Estados Unidos de América[4],[18].

En 1946 crearía la primera Unidad de Resucitación en el Instituto de Cirugía Cardiovascular A.N. Bakulev. En compañía de Naum L Gurvitch estudiarían los problemas de conducción eléctrica del corazón, como la fibrilación ventricular, demostrando en estudios en perros que ésta se podría revertir con una carga de impulso externa, utilizando una corriente continua de contragolpe, lo que haría posible los primeros desfibriladores portátiles. En Norteamérica, Hooker, Kouwenhoven y Langworthy habían realizados estudios financiados por el Instituto Eléctrico Edison y el Instituto Nacional de Salud en Estados Unidos, con el fin de desarrollar un desfibrilador para los empleados de las empresas de energía quienes sufrían electrocución frecuente; el primer informe de desfibrilación exitosa lo hizo Claudio S. Beck en 1947 con un paciente a quien se le había realizado una cirugía cardiaca[20]. Negovsky creó en 1959 el primer centro para tratamiento de shock y estados terminales en el Hospital Municipal S. P. Botkin, y en 1962[4],[18] la primera unidad de resucitación, al igual que la UCI[4]. En 1958 el senador estadounidense Hubert Humphrey realizó una visita al laboratorio de Negovsky, por lo que más tarde en uno de sus discursos instaría a los científicos norteamericanos a investigar sobre la fisiología de la muerte y su recuperación, con el ánimo de competir en el contexto de la guerra fría con los avances científicos gestados desde la Unión Soviética[18].

\section{Discusión}

"Today anesthesia, tomorrow the world". Peter Safar[3].

Pese a las tensiones ideológicas, económicas y políticas por la hegemonía mundial entre los Estados Unidos de América (bloque occidental-capitalista) y la Unión Soviética (bloque del este-comunista), durante la guerra fría, el trabajo de Safar y Negovsky demuestra que los flujos de información y la construcción colectiva del conocimiento deben estar por encima de las fronteras políticas.

Esto se evidencia en el artículo escrito por Safar sobre Negovsky, publicado en 2001, dos años antes de la muerte de ambos personajes. Allí Safar reconoce los aportes de Negovsky y los lazos de amistad que los unieron desde 1962, y las contribuciones al conocimiento científico en reanimación que ambos hicieron gracias a una comunicación y colaboración científica permanente, así como múltiples encuentros en eventos internacionales, que permitieron hacer un eco el uno del otro, de los avances realizados para "restaurar socialmente a una persona víctima de un paro cardiaco"[4],[6]. Safar reconoce que Vladimir Negovsky pasará a la historia de la medicina como el padre de la ciencia de la medicina de la reanimación o reanimatología. Precisa Safar que, gracias a los estudios del investigador soviético, se han podido comprender los procesos fisiopatológicos que se producen en la muerte clínica y las medidas que se pueden instaurar en la resucitación de emergencia para preservar la supervivencia sin daño cerebral. Muchos han sido los científicos de diversos países que se han inspirado en estos conocimientos para desarrollar lo que hoy se conoce como el quinto eslabón de la cadena de supervivencia, el manejo integral posparo cardiaco[4],[6]. Negovsky introdujo conceptos como el de estado terminal, muerte clínica (período de ausencia de flujo de sangre que es potencialmente reversible) y muerte biológica (paro cardiaco con muerte cerebral irreversible); también realizó investigaciones alrededor de la asfixia en el recién nacido, la desfibrilación con onda eléctrica bifásica (incluso 30 años antes que fuera un tema común en el mundo occidental), desastres civiles y rescate, lesiones por choques eléctricos, compresiones torácicas externas (1940), tratamiento de la exanguinación y de la hemorragia, hipotermia terapéutica (1960), abordaje del infarto miocárdico, experiencias cercanas a la muerte, donación de órganos, accidentes en embarazadas, shock y trauma[4],[19].

Hablar en términos de reanimación más que de resucitación y de reanimatología como ciencia, se lo debemos a Negovsky y a Safar. El sufijo "anima" referente a la mente o al espíritu, indicaría el esfuerzo que se hace en la resucitación por la sobrevivencia sin daño cerebral[4]. Como ciencia, la reanimatología incluye aspectos relacionados al control de la fisiopatología de la coagulopatía vascular, la ventilación, 
las arritmias, los cambios hemodinámicos y de temperatura, las anormalidades metabólicas, la isquemia residual y la inestabilidad eléctrica periparo[4],[19]. Negovsky inició la resucitación en el laboratorio, implementando novedosos métodos de reanimación como la administración de sangre intraarterial en el shock hemorrágico letal[3].

Safar le dio importancia a la falta de oxigenación en las condiciones de paro cardiaco y fue quien implementó la ventilación con presión positiva entre las compresiones cardiacas externas, que habían sido redescubiertas por los doctores Guy Knickerbocker, James Jude y William Kouwenhoven en 1957[21],[22]. (En 1861 se había introducido por primera vez la técnica de compresión torácica en decúbito supino con los brazos levantados)[2], y las aplicó en forma de nemotecnia a la secuencia de la reanimación, estructurando el $A B C$ (hoy CABD primario), el DEF (hoy $A B C D$ secundario) y el GHI (hoy manejo integral posparo cardiaco), los cuales se debían hacer en el espacio prehospitalario, hospitalario y en la unidad de cuidado intensivo respectivamente. Safar, además, implementó la maniobra que permite permeabilizar la vía aérea, o triple maniobra que consiste en extender la cabeza, abrir la boca y subluxar la mandíbula; y la primera unidad en cuidados intensivos en compañía del intensivista Max Harry Weil y el cirujano William Shoemaker en la década de 1960 y el primer servicio de emergencias paramédicas[5],[15],[22], en un esfuerzo de llevar las técnicas de anestesia usadas en el quirófano a las calles para salvar vidas, así como la importancia de la anestesiología en la cirugía y en la reanimación para garantizar la sobrevida del paciente; la premisa de Safar era: "surgery would not advance without better life support and you learn life support in anesthesiology" ("la cirugía no avanzaría sin un mejor soporte vital y usted aprende el soporte vital en anestesiología"[3]).

Safar junto con el doctor Hugh Rosomoff en 1950, sugirieron el uso de la hipotermia moderada en el manejo de lesiones cerebrales por isquemia y trauma[14],[21],[22], por lo que Safar implementó el uso clínico de la misma en la resucitación cerebral[3],[23],[24]. Uno de sus pupilos, Fritz Sterz en Viena, continuó investigando el rol del uso clínico de la hipotermia en la reanimación cerebral y dirigió un estudio clínico multicéntrico en Europa con resultados satisfactorios, al demostrar que después de un paro cardiaco con fibrilación ventricular, la hipotermia terapéutica leve mejora los resultados neurológicos de los pacientes[3],[25]. El uso de la hipotermia también tomó otro rumbo, al aplicarla en forma profunda, con el fin de controlar la hemorragia y mantener vivo co- razón y cerebro (animación suspendida)[3],[26],[27].

A partir del conocimiento del trabajo de James Elam sobre la ventilación para mantener la gasometría sanguínea normal, Safar promulgó la importancia de la ventilación, proponiendo en la reanimación la ventilación boca a boca en 1958; esta maniobra se posicionó como un método superior a la elevación de brazos con contrapresión sin un tubo traqueal (Método de Holger Nielsen), ya que la respiración boca a boca con una adecuada técnica podría oxigenar de forma satisfactoria, y producir la salida del $\mathrm{CO}_{2}$, siendo un método fácil de aprender por un lego, y de utilidad para el rescate de adultos y niños[28],[29]. El mismo, aunque tuvo un impacto mundial, fue rechazado por Lloyds de Londres[15].

En una de sus presentaciones en la Sociedad Escandinava de Anestesiología, Safar conoció en 1958 al doctor Bjorn Lind y a Asmund Laerdal, un fabricante de muñecos. Interesados en el tema de la reanimación, surgió la idea de crear el maniquí de entrenamiento llamado "Resusci Anne" en 1960[5],[15]. El rostro del maniquí Resusci Anne no es cualquier rostro, éste representa la imagen de una joven suicida cuyo cuerpo había sido recuperado del río Sena y expuesto en una funeraria de París para que de los transeúntes que pasaban por allí, alguno pudiera reconocerla. Fue un patólogo quien, al verla, sintió una fascinación por su cara y sonrisa apacible, por lo que pidió a un fabricante de máscara, que le hiciera un molde en yeso, y así esta máscara mortuoria fue llamada "L'Inconnue de la Siene" (la desconocida del Sena) que inspiró no solo a novelistas, poetas y pintores, sino también a estos pioneros de la simulación y la reanimación cardio cerebro pulmonar[30],[31]. (Figura 2).

Safar y Ake Grenvik desarrollaron las primeras guías en Estados Unidos sobre evaluación de muerte cerebral, tópico también investigado por Negovsky. Esto dinamizó las posibilidades de la cirugía de trasplante, que con Thomas Starzl, médico llamado el padre del trasplante moderno, tomaron un auge inusitado en Pittsburg, pues él sería el primero en trasplantar un hígado humano, además de haber implementado el uso de la ciclosporina y el tacrolimus, y de haber desarrollado las técnicas avanzadas de preservación de órganos. Safar y Grenvik, junto con Alan Meisel, bioeticista y abogado, diseñaron además las primeras guías para la terapia en soporte vital en caso de pacientes desahuciados[3].

El Laboratorio de Reanimatología General de Negovsky en Moscú (Figura 3), serviría de modelo para que Safar estableciera el IRRC (Centro de Investigación en Resucitación de la Universidad de Pittsburg). 


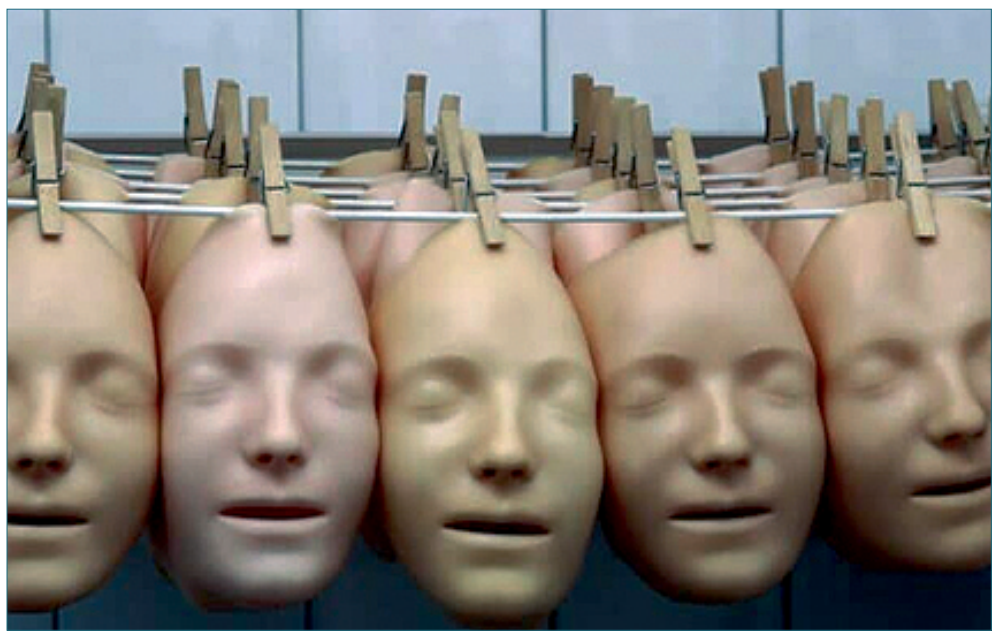

Figura 2. El rostro de La Desconocida del Sena para maniquíes de RCP30.

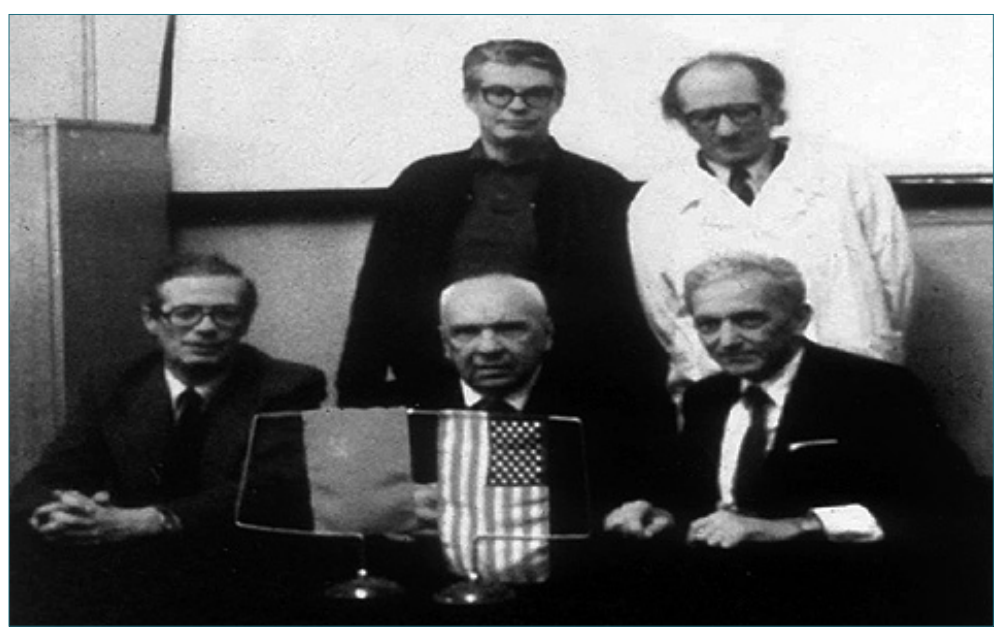

Figura 3. Laboratorio en Moscú de Vladimir Negovsky, 1973: de pie de izquierda a derecha A. Gurvich y Tabak. Sentados de izquierda a derecha, Kassil, Negovsky y Safar[4].

En este centro Safar trabajaría durante 15 años con Norman Abramson y emprendería el ensayo clínico de resucitación cerebral, el cual se realizó en 20 centros médicos de siete países. Este centro fue renombrado en honor a Safar, "The Safar Center for Resuscitation Research", y continuó bajo el liderazgo de Patrick Kochanek. En la década de 1970 Safar y Negovsky con Rudolf Frey crearon la Asociación Mundial de Medicina de Desastres y Emergencias WADEM (World Association for Disaster and Emergency Medicine), y promovieron también el movimiento médico internacional para la prevención de la guerra nuclear[8]. Safar le dio a Frey todo el crédito de la creación de esta asociación que se inauguró en Mainz en septiembre de 1977 en el primer Congreso Mundial sobre Medicina de Emergencias y Desastres[32]. Safar, Negovsky y
Frey estuvieron interesados en pertenecer a organizaciones internacionales desde las cuales pudieran agenciar cambios y dar directrices sobre la medicina de desastres, el cuidado crítico, la reanimatología y lo que ellos llamarían la medicina de la paz. Luchar contra la muerte en cerebros demasiado jóvenes para morir, caracterizar los escenarios críticos en que el paciente se debate por la vida, entenderla, definirla, crear estrategias para evitarla y disminuir el daño cerebral.

La muerte biológica definida por Negovsky como la ausencia de funcionamiento del cerebro, o lesión cerebral irreversible e incompatible con la vida, distinta de la muerte clínica cuando hay paro y ausencia de pulso, daba a ésta la posibilidad de retorno, siempre y cuando se iniciara una reanimación apropiada y precoz. El último encuentro cara a cara de los dos titanes 
de la reanimación fue en 1999 donde Safar visitó a Negovsky en su cumpleaños número 90. La muerte del uno presagiaría la del otro, pues finalmente Vladimir Negovsky moriría el 2 de agosto de 2003 y un día después Peter Safar, cada uno en el país que vio forjar sus vidas entregadas a la labor médica[3],[4],[5].

\section{Conclusión}

Los avances en el campo de la reanimatología y la medicina crítica tuvieron dos adalides en el mundo, un médico anestesiólogo nacido en Austria, Peter Safar, y un médico ruso, fisiopatólogo nacido en Ucrania[33]. Se establece una concepción del trabajo científico como un proceso social e investigativo, el cual tiene un componente individual que no puede ser soslayado, bajo el cual se ejerce un liderazgo dentro de una colectividad científica para llegar final- mente a uno o varios productos que transforman la vida humana, en este caso los procesos de la vida y la muerte. Para llegar a ser realmente grande hay que estar a la altura de la gente decía el barón de Montesquieu, y sólo es posible avanzar cuando se mira lejos, decía el filósofo español José Ortega y Gasset. Estos dos hombres trabajaron en sus laboratorios para la gente, pensando en el bien común y adelantándose a la época, sin ningún asomo de egoísmo, y a fe que llegaron lejos y desarrollaron lo que hoy se conoce como la ciencia de la reanimación o reanimatología.

\section{Agradecimientos}

A la Universidad Nacional de Colombia y a la Universidad de Antioquia.

Conflicto de Interés: ninguno.

Financiación: ninguna.

\section{Referencias}

1. Journal of Pearls in Intensive Care Medicine. Herrero-Varon's MD Editors. Gijon (Asturias, Espana) and Houston (TX, USA). Languaje EN/ES 2011-2014. Revisado el 14 de diciembre de 2018 https://archive.org/ stream/echanischeReanimatio nssysteme/B \%29\%200.3\%20 Historia\%20de\%20la\%20 Reanimaci\%C3\%B3n\%20 cardiopulmonar_djvu.txthttps:// archive.org/stream/echanischeR eanimationssysteme/B\%29\%20 $0.3 \% 20$ Historia $\% 20$ de $\% 20$ la\%20Reanimaci\%C3\%B3n\%20 cardiopulmonar_djvu.txt

2. Temas selectos de reanimación cerebro cardiopulmonar. Editorial UN. Editor Navarro Vargas JR. Bogotá Colombia, agosto 2015. ISBN 978-958-775-445-2 (e-book)

3. Grenvik A, Kochanek PM. The incredible career of Peter J. Safar, MD: the Michelangelo of acute medicine. Crit Care Med. 2004 Feb;32(2 Suppl):S3-7. https://doi.org/10.1097/01. CCM.0000110733.48596.4F PMID:15043225

4. Safar P. The resuscitation greats. Vladimir Negovsky the father of 'reanimatology'. Resuscitation. 2001;49:2239. https://doi.org/10.1016/ S0300-9572(01)00356-2 PMID:11723996

5. Acierno LJ, Worrell LT. Peter Safar: father of modern cardiopulmonary resuscitation. Clin Cardiol. 2007 Jan;30(1):524. https://doi.org/10.1002/ clc.20042 PMID:17262769

6. Negovsky VA. The second step in resuscitation-the treatment of the 'post-resuscitation disease'. Resuscitation. 1972 Mar;1(1):17. https://doi.org/10.1016/03009572(72)90058-5 PMID:4653025

7. Safar P. "the Father of CPR" is dead at 79. The New York Times, archives, august 6, 2003. Consultado 2 de diciembre de 2018 https://www.nytimes. com/2003/08/06/us/peter-safarthe-father-of-cpr-is-dead-at-79. html

8. Klain M. Obituary Vladimir Alexandrovitch Negovsky. Resuscitation. 2003;59(1):7-9. https:// doi.org/10.1016/j.resuscitation.2003.08.010.

9. Oransky I. Obituary. Peter Safar. Teh Lancet; 2003. p. 362.

10. Mitka M, Peter J. Safar, "Father of CPR," Innovator, Teacher, Humanist. Medical News \& Perspectives. JAMA. 2003;289(19):24856. PMID:12759308

11. Navarro-Vargas JR, Díaz JL. Sindrome posparo cardiaco. Rev Colomb Anestesiol. 2014;42(2):107-13. https://doi. org/10.1016/j.rca.2014.01.001.

12. Hobsbawm E. Historia del siglo XX. Buenos Aires: Editorial Crítica; 1998.

13. Brannigan A. The social basis of scientific discoveries. Cambridge: Cambridge University Press; 1981.

14. Hidalgo C, Schuster F. El descubrimiento científico como fenómeno comunitario. Cuad Antropol Soc. 2003;(18):67-77.

15. Kochanek PM, Grenvik 
A. A tribute to Peter J.

Safar, MD. Crit Care Med.

2003 Nov;31(11):2571-3.

https://doi.org/10.1097/01. CCM.0000100838.26307.B0 PMID:14605525

16. Safar P, Elam J. Advances in cardiopulmonar resuscitation. New York: Springer-Verlag; 1977. https://doi.org/10.1007/978-14612-6338-8.

17. Rubertsson S, Safar P. Reanimación cardiopulmonar y cerebral. Sección I. En Reanimación y emergencias médicas. Edit Shoemaker WC, Ayres SM. Pp 11-21. http://media.axon.es/ pdf/49906.pdf revisado 4 de enero de 2019.

18. Moroz, V. Poccññckr peahhmatojiorhr ceromhr (Reanimatología Hoy) Pocchhckar akamemhr hayk, mpeehhhym, «Hayka B Pocchh», 2014 (Academia Rusa de Ciencia, Presidium, "La ciência em Rusia"); 2(200): 13-18.

19. Chamberlain D. Vladimar Negovsky: The father of Reanimatology. The Negovsgy Memorial Lecture, Given at The Seventh Scientific Congresso of the European Resuscitation Council, Budapest September 2004. OBLLLAR P EAHHMATOJIOLHR (Reanimatología General), 2005; I:(2): 5769. https://www.researchgate. net/publication/279234006_Vladimar_Negovsky_The_Father_of_ Reanimatology

20. Navarro-Vargas JR, Muñoz R. Historia de la terapia eléctrica en reanimación. Rev Cub Anes y Rean. 2017;16(1):1-9.

21. Acosta P, Varon J, Sternbach
GL, Baskett P, Kouwenhoven W, Jude J, et al. Resuscitation great. Kouwenhoven, Jude and Knickerbocker: the introduction of defibrillation and external chest compressions into modern resuscitation. Resuscitation. 2005 Feb;64(2):13943. https://doi.org/10.1016/j. resuscitation.2004.12.004 PMID:15680520

22. Huertas-Torrijos J, Díaz B, GarcíaMartínez SA. Reanimación cardiopulmonar y cerebral. Historia y desarrollo. Rev Asoc Mex Med Crit Ter Intensiv. 2001;15(2):5160.

23. Safar PJ, Kochanek PM. Therapeutic hypothermia after cardiac arrest. N Engl J Med. 2002 Feb;346(8):6123. https://doi.org/10.1056/ NEJM200202213460811 PMID:11856801

24. Parellada Blanco J. Peter Josef Safar "La resucitación cardiopulmonar y cerebral es para aquella persona con un corazón y un cerebro lo suficientemente buenos como para que mueran". Rev Cub Med Int y Emerg. 2003;2(3):1.

25. Mild Therapeutic Hypothermia to Improve the Neurologic Outcome after Cardiac Arrest. New England Journal of Medicine [Internet]. Massachusetts Medical Society; 2002 Feb 21;346(8):549-56. Available from: http://dx.doi.org/10.1056/ nejmoa012689

26. Ravitch MM, Lane R, Safar $P$, Steichen FM, Knowles $P$. Lightning stroke. Report of a case with recovery after cardiac massage and prolonged artificial respiration. N Engl J Med. 1961 Jan;264(1):368. https://doi.org/10.1056/ NEJM196101052640109 PMID:13739911

27. Liu Y, Li S, Li Z, Zhang J, Han JS, Zhang $Y$, et al. A safety evaluation of profound hypothermiainduced suspended animation for delayed resuscitation at 90 or 120 min. Mil Med Res. 2017 May;4(1):16-22. https://doi. org/10.1186/s40779-017-01274 PMID:28573043

28. Safar P. Ventilatory efficacy of mouth-to-mouth artificial respiration; airway obstruction during manual and mouth-to-mouth artificial respiration. J Am Med Assoc. 1958 May;167(3):335-41. https://doi.org/10.1001/ jama.1958.72990200026008c PMID:13538712

29. Baskett PJ, Safar P. The early years 1924-1961, the birth of CPR, Resuscitation. ERC. 2001;50:17-22.

30. A compendium photographs L'inconnue de la Siene. Best New Poets 2012. http://www. chuckcarlise.com/wp-content/ uploads/2011/09/COMPENDIUM-OF-PHOTOGRAPHS.pdf

31. La mujer desconocida más famosa del mundo. https:// hipertextual.com/2016/03/ladesconocida-del-sena

32. Frey R. In memoriam. Safar P. Crit Care Med. 1982;10(6):421.

33. Vladimir A. Negovsky. Safar P. Resuscitation. 1994;28:175-7. 